\section{Alpha control: Effectiveness of two kinds of feedback*}

\author{
DAVID HORD and JOSEPH BARBER \\ Navy Medical Neuropsy chiatric Research Unit, San Diego, Calif. 92152
}

Eleven volunteer Ss participated on 2 separate days in an alpha control study. On Day 1, an auditory feedback tone was on during the presence of alpha and $S$ was instructed to turn alpha on or off during alternating 8-min feedback sessions. Preceding each feedback session, $\mathrm{S}$ was instructed to turn alpha on or off for $8 \mathrm{~min}$ without the feedback tone. On Day 2, the same procedure was followed, except that tone-on meant a low level of alpha and tone-off meant a high level of alpha. Alpha control, defined as the percent alpha during the alpha-on session minus the percent alpha during the alpha-off session, was significant on both days and was positively correlated with baseline percent alpha. Feedback sessions showed significant appropriate changes compared to no-feedback sessions only during the first sessions of Day 1 . Heart rate was not significantly related to percent alpha.

In everyday experience, people are not aware of the "state" of their EEG, alpha, or of any brain wave. Through alpha feedback training, the individual is made aware of the presence of alpha by hearing or seeing a contingent signal. Individuals presumably learn to alter their subjective "state" in order to generate alpha for greater and more frequent lengths of time. The individual becomes aware of a difference in his subjective feeling when the feedback indicates that his alpha is present, and eventually he can "turn on alpha" without the aid of the feedback.

Experiments involving alpha feedback have been accumulating since Adrian \& Mathews's (1934) report of a correlation between a subjective impression of looking or not looking and the absence or presence of the alpha rhythm in the EEG. More recently, Kreitman \& Shaw (1965) found evidence for alpha enhancement in seven Ss doing mental tasks-tasks traditionally considered "alpha blockers." The operant technique, using a feedback paradigm in which a visual or auditory signal serves as a positive reinforcer, does not require a particular way to enhance alpha and has been shown to be effective in alpha control (e.g., Nowlis \& Kamiya, 1970; Brown, 1970). Subjective reports obtained in these studies indicate that a wide range of methods are effective for increasing or decreasing alpha. Voluntarily

*This research was supported by Department of Defense Advance Research Projects Order 1596, Program Codes 0D20 and 1D20. The opinions or assertions contained herein are the private ones of the authors and are not to be construed as official or as necessarily reflecting the views of the Department of the Navy. The authors thank Ardie Lubin, Laverne Johnson, and Paul Naitoh for helpful comments on all aspects of the study. enhanced alpha has also been attributed to "reduced inhibition" as opposed to operant conditioning (Lynch, Orne, Paskewitz, \& Costello, 1969) and to classical conditioning (Cleeland, Booker, \& Hosokawa, 1970).

The purpose of the present experiment was to (1) replicate those studies reporting rapid alpha control with a contingent auditory feedback signal, (2) test whether or not alpha control will continue without the feedback, (3) test whether or not feedback where tone-on accompanies high alpha is any better than feedback where tone-off accompanies high alpha, and (4) study the relation of heart rate (HR) to alpha. Four hypotheses have been formulated and tested:

\section{Hypothesis 1}

Volunteer Ss will show enhanced alpha when instructed to keep alpha on, using a contingent feedback tone as a guide, and will show diminished alpha when instructed to keep alpha off, using the feedback tone as a guide. Hypothesis 2

Following the first alpha on session with feedback, alpha control, defined during the alpha on and alpha off sessions, will be no greater under the feedback condition than under the no-feedback condition.

\section{Hypothesis 3}

Alpha control, as defined above, accompanies alpha as it is when tone-off accompanies alpha.

$$
\text { Hypothesis } 4
$$

Mean HR levels will be positively correlated with percent-alpha, as suggested by Lacey (1967).

\section{SUBJECTS}

Sixteen volunteer male and female Ss from local colleges (age range: 19-35) agreed to come to the as the difference between alpha levels will be the same when tone-on laboratory on at least two separate occasions to take part in an "alpha feedback experiment." The records of 11 Ss were retained on the basis of completeness and freedom from extensive artifact.

\section{APPARATUS}

EEG $\left(\mathrm{O}_{\mathrm{z}}-\mathrm{A}_{2}\right)$, filtered EEG (through a Krohn-Hite Model 330 bandpass filter, set at $10 \mathrm{~Hz}$ ), a relay state indicator to show when the feedback circuit was enabled, EOG, finger pulse, skin potential from the palmar surface of the left hand, HR (through a Beckman 9857 cardiotachometer), and respiration were recorded on a Beckman Type $R$ dynograph. The filtered EEG was amplified by a Tektronix 2A61 differential amplifier and rectified. The output of the rectifier entered a Schmitt trigger, and, subsequently, a BRS relay and audio generator provided the feedback tone. A Hewlett-Packard Model 5321 B electronic counter, activated by the same relay closure that enabled the feedback circuit, counted the time the relay was closed to the nearest 10 th of a second.

\section{PROCEDURE}

Each experimental day included (1) attachment of electrodes and transducers, (2) brief discussion of the general purpose and nature of the experiment ( $S$ was encouraged to move as little as possible and to keep his eyes open at all times during the experiment), (3) brief period of familiarization with the feedback signal (S closed his eyes temporarily while the pitch and loudness of the tone were modified as necessary to provide a feedback signal that was not aversive but clearly discernible), and (4) instruction of $\mathrm{S}$ to open his eyes and a trial-and-error adjustment of the amplifier in the feedback so that the relay would be enabled approximately $20 \%$ of the time, when $S$ was resting. (Our intention was to equalize, across Ss, the baseline "alpha level," independent of each S's absolute abundance of alpha.)

Each 8-min session was initiated by a brief explanation and appropriate set of instructions from the $E$ : (1) Baseline $(B L)$. S is instructed to sit quietly for $8 \mathrm{~min}$. (2) Alpha on, no feedback $(O N, N F B)$. $S$ is instructed to try to produce alpha activity on the basis of whatever understanding he might have about alpha. (3) Alpha on with feedback (ON,FB). $\mathrm{S}$ is instructed to keep alpha on as much as possible, using the contingent feedback tone as a guide. (4) Alpha off, no feedback $(O F F, N F B) . \mathrm{S}$ is instructed to keep alpha off, on the basis of whatever understanding he might now have about alpha. (5) Alpha off, with feedback $(O F F, F B) . S$ is instructed to 
Table 1

Percent Alpha for the Baseline-Recovery Sessions, On vs Off Instructions, and the Feedback va No-Feedback Conditions on Day 1 and Day 2

\begin{tabular}{|c|c|c|c|c|c|}
\hline \multirow[b]{2}{*}{ Session } & \multirow{2}{*}{$\begin{array}{l}\text { Baseline- } \\
\text { Recovery } \\
\text { (1 and } 8 \text { ) }\end{array}$} & \multicolumn{2}{|c|}{ Turn Alpha Off } & \multicolumn{2}{|c|}{ Turn Alpha On } \\
\hline & & $\begin{array}{c}\text { NFB } \\
\text { (4) }\end{array}$ & $\begin{array}{l}\text { FB } \\
\text { (5) }\end{array}$ & $\begin{array}{c}\text { NFB } \\
\text { (6) }\end{array}$ & $\begin{array}{c}\text { FB } \\
(3 \text { and } 7)\end{array}$ \\
\hline \multicolumn{6}{|c|}{ Day 1} \\
\hline VAC & 42.0 & 50 & 25 & 76 & 65.5 \\
\hline GB & 13.5 & 9 & 2 & 18 & 11.0 \\
\hline $\mathbf{E M}$ & 20.5 & 21 & 4 & 9 & 40.0 \\
\hline IA & 14.5 & 9 & 5 & 8 & 23.5 \\
\hline GW & 20.5 & 19 & 19 & 42 & 24.0 \\
\hline MH & 34.0 & 9 & 9 & $\mathbf{3 9}$ & 36.5 \\
\hline TD & 27.5 & 8 & 4 & 40 & 29.5 \\
\hline $\mathbf{K J}$ & 5.5 & 5 & 2 & 17 & 36.0 \\
\hline $\mathbf{M A}$ & 20.5 & 8 & 10 & 60 & 41.0 \\
\hline $\mathrm{LM}$ * & - & 18 & 16 & 15 & 12.5 \\
\hline VA & 27.0 & 7 & 1 & 28 & 32.0 \\
\hline $\begin{array}{l}\text { Mean } \\
\text { SD }\end{array}$ & $\begin{array}{l}22.55 \\
10.56\end{array}$ & $\begin{array}{l}14.82 \\
12.87\end{array}$ & $\begin{array}{l}8.82 \\
7.96\end{array}$ & $\begin{array}{l}32.00 \\
21.83\end{array}$ & $\begin{array}{l}31.95 \\
15.06\end{array}$ \\
\hline \multicolumn{6}{|c|}{ Day 2} \\
\hline VACt & - & - & 一 & - & - \\
\hline GB & 7.0 & 7 & $\mathbf{1}$ & 29 & 28.0 \\
\hline EM & 15.5 & 2 & 1 & 15 & 18.0 \\
\hline IA & 25.0 & $\mathbf{3 1}$ & $\mathbf{9}$ & 54 & 42.5 \\
\hline GW & 6.0 & 11 & 15 & 31 & 22.5 \\
\hline MH & 6.5 & 20 & 21 & 13 & $\mathbf{3 1 . 0}$ \\
\hline TD & 22.5 & 10 & 9 & 35 & 22.5 \\
\hline $\mathbf{K J}$ & 32.5 & 15 & 18 & 14 & 69.5 \\
\hline MA & 14.0 & 19 & 12 & 27 & 30.5 \\
\hline $\mathbf{L M}$ & 17.5 & 24 & 16 & 21 & 22.0 \\
\hline $\mathbf{V A}$ & 41.0 & 49 & 62 & 74 & 73.5 \\
\hline Mean & 18.75 & 18.80 & 16.40 & 31.30 & 36.00 \\
\hline SD & 11.66 & 13.60 & 17.35 & 19.38 & 19.93 \\
\hline
\end{tabular}

*S LM misunderstood baseline instructions; data is not included in baseline comparisons. +S VAC data available for Day 1 only; not included in Day 1 us Day 2 comparisons.

keep alpha off, using the tone as a guide. (6) Alpha on, no feedback $(O N, N F B)$. Same as Session 2. (7) Alpha on, with feedback (ON,FB). Same as Session 3. (8) Recovery (R). Same as Session 1.

The procedure described above was followed on Day 1. On Day 2, the same procedure was followed, except that the tone was off when alpha appeared. The $S$ was told to control alpha and to use the tone as a guide, but that now tone off meant alphe present, and conversely.

QUANTIFICATION OF DATA

Cumulative alpha time was read to the nearest second from the Hewlett-Packard counter at 30-sec intervals for each 8-min session. After each session, the counter was reset to zero and a cumulative record for the next 8-min session was started. HR was read for the first five beats at the end of each 30-sec epoch. The average HR for a given 8-min session is the mean of 16 sample means, each sample mean being associated with a corresponding measure of cumulative alpha time.

\section{RESULTS}

The results of the experiment on which tests of the first three hypotheses are based are given in Table 1. BL (Session 1) and $R$ (Session 8) were combined. The
( $t=1.00$ on Day 1 and 1.30 on Day 2). Alpha control was as good without the feedback as it was with the feedback.

\section{Hypothesis 3}

Can Ss control alpha levels independently of whether tone-on or tone-off is used as feedback? The same measure of alpha control was used as in Hypothesis 2 (Sessions 7 and 3), minus Session 5 for the feedback condition). The mean alpha control was 23.1 on Day 1 (tone-on accompanies alpha) and 19.6 on Day 2 (tone-off accompanies alpha). A t test indicated no difference between the 2 days. Tone-on as feedback for alpha enhancement is as effective as tone-off.

\section{Hypothesis 4}

On each day, for each $S, H R$ and percent alpha were obtained for each session, eight sessions in all. The product-moment correlation between $H R$ and percent alpha was calculated for each $S$. These correlations, listed in Table 2, tend to be similar within a $S$ (suggesting some relation between the variables), but the average does not differ significantly from zero (indicating that the relationship is not the same across Ss, if there is any relationship at all).

There was a significant positive correlation of about .60 between alpha control (ON minus OFF) and the $B L$ alpha level. A rather wide distribution of percent alpha levels for the $B L$ session occurred despite attempts to adjust each person's threshold such that he would receive $20 \%$ feedback. The distribution ranged from $7 \%$ to over $50 \%$. The regression of the alpha control measure on BL percent alpha is shown for Days 1 and 2 in Fig. 1.

$$
\text { DISCUSSION }
$$

The results of the experiment are generally consistent with those reported by Nowlis \& Kamiya (1970); both alpha enhancement and suppression are possible in volunteer Ss. In the present experiment, control was better for the ON condition than

Table 2 significantly less than the average percent alpha during $B L$ and $R$ sessions ( 1 and 8$)$ on Day $1(t=5.27$, $p<.01)$; there was no significant difference on Day $2(t>1.0)$.

$$
\text { Hypothesis } 2
$$

Our measure of alpha control without feedback is the percent alpha during the ON,NFB session (6), minus the percent alpha during the OFF,NFB session (4). Our corresponding measure of alpha control with feedback is the percent alpha during the ON,FB sessions ( 7 and 3 ), minus the percent alpha during the OFF,FB session (5). These two measures did not differ significantly for either day of Heart-Rate Level and Linear Correlation of Heart-Rate Lev
Percent Alpha for Each $S$ on Day 1 and Day 2

\begin{tabular}{lrr}
\hline & \multicolumn{2}{c}{$r$} \\
\cline { 2 - 3 } S & Day 1 & Day 2 \\
\hline GB & .11 & .30 \\
EM & .47 & .12 \\
IA & -.84 & -.06 \\
GW & -.84 & -.47 \\
MH & -.39 & -.13 \\
TD & .51 & .02 \\
KJ & .52 & .74 \\
MA & .55 & -.20 \\
LM & .34 & .00 \\
VA & -.01 & -.14 \\
\hline
\end{tabular}


for the $O F F$ condition. There was no significant alpha suppression during the OFF condition on Day 2. Part of the inability to suppress alpha on Day 2 may be due to the procedure used for determining the threshold of the feedback device (rather than to limitations of alpha control). In the present experiment, the intensity of EEG alpha which triggered the relay was set so that the relay was closed approximately $20 \%$ of the time during $\mathrm{BL}$. The five Ss who were below $20 \%$ during $\mathrm{BL}$ found it very difficult to reduce their alpha further. The optimal level of feedback for alpha control in humans has not been determined, but it appears to be an important variable.

It seems clear that the degree to which an individual can demonstrate control of his alpha is closely dependent on the threshold level initially set for him. If an individual begins the $\mathrm{BL}$ session with the relay closing $20 \%$ of the time, then he has a rather wide range of improvement possible to him in subsequent feedback sessions. If he begins the BL session with the relay closing $50 \%$ of the time, his range for subsequent improvement is comparatively limited. This reasoning follows from the premise that little or no learning will result when the tone is on (or off) $100 \%$ of the time.

The issue of the appropriate threshold level is largely settled by the purpose of the training. If the object of the training is to turn alpha off (and presence of the tone means presence of alpha), then the higher the threshold level, the more learning is possible. (Eighty percent during BL would then be a good level for turning alpha off.) If the same training were to use the presence of the feedback tone to mean absence of alpha, the same procedure would of course be employed. The data of Fig. 1 make it clear that those $S s$ who did receive the higher BL levels (due to our inability to adjust the level precisely to $20 \%$ ), tended also to show the greatest degree of control, as it was defined here.

If alpha training is to include both turning alpha on and off (using the same design as in the present study), then probably a $50 \%$ level is best. However, it would seem that optimal control would result from a different design, one that would allow for changing the threshold level between the two conditions (say, 20\% for alpha on and $80 \%$ for alpha off).

Alpha control during feedback sessions was not significantly greater than alpha control during no-feedback sessions. This conclusion is based on our measure of alpha control (which is the difference between percent alpha

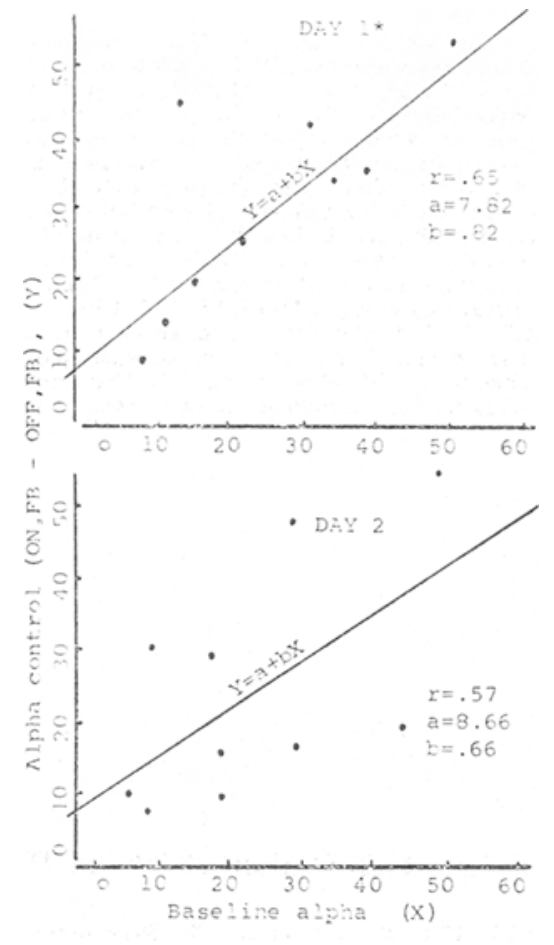

Fig. 1. Linear regression of alpha control on threshold level during baseline. (S LM misunderstood instructions for baseline and is not included here.)

during $O N$ and OFF sessions). Other comparisons of feedback vs no-feedback can be made within the ON and OFF sessions, and they generally indicate that feedback is better than no feedback. [Compare Sessions 4 and 5 for the OFF condition and Sessions 6 and ( 3 and 7 ) for the ON condition shown in Table 1.] But only one of these comparisons is significant: Sessions 4 and 5 on Day 1 , which includes the first exposure to the feedback signal. Apparently this initial $16-\mathrm{min}$ period, which includes both feedback and no-feedback conditions, is sufficient to increase the effectiveness of the no-feedback situation. Brown (1970) has also concluded that rapid alpha control can occur within the first practice session.

The test of Hypothesis 3 showed that the measure of alpha control was no better for the tone-on alpha present feedback than for the tone-off alpha present feedback (Day 1 vs Day 2). Possibly tone-off alpha present feedback will be useful for long-term high-alpha training, since presence of a constant tone over long periods of time could lead to auditory fatigue or habituation. A disadvantage of this type of feedback is indicated in S's reports that, after a prolonged absence of the tone (presence of alpha), they could easily forget the nature of the task, the importance of keeping their eyes open, etc. With the tone-on alpha present feedback, Ss were constantly "reminded" of the nature of the task, etc.

It is possible that the lack of support for a hypothesized relationship between alpha level and $H R$ level is due to inappropriate sampling techniques. Analysis of data in subsequent studies continues to indicate a correlation between these two variables during certain, as yet unspecifiable, periods.

The important question of what is occurring at the level of neural activity in the brain during alpha regulation can be discussed only within the limits set by our knowledge of the intrinsic rhythms involved. One point of view, which is based on extensive experimental work with animals, has recently been summarized by Andersen \& Andersson (1968). Their theory of a facultative pacemaker at the level of specific and association nuclei in the thalamus views these structures as the primary synchronizing mechanism of alpha waves. Intrathalamic intrinsic rhythms, which are postulated to be a function of the inhibitory properties of the thalamic cells involved, modulate thalamocortical projection system neurons at a rate that corresponds to the alpha range of frequencies. Synchronization, which, in the present context, corresponds to sustained alpha activity, is thus a thalamic rather than a cortical phenomenon and, as such, occurs when peripheral sensory inputs are minimal. This interpretation is generally consistent with the subjective state that is usually associated with high alpha activity: passive, attending to internal as opposed to external stimuli, etc.

\section{CONCLUSIONS}

(1) Voluntary increases or decreases in alpha are possible in Ss with eyes open. In this study, where the threshold was adjusted to enable the relay about $20 \%$ of the time during the baseline, the Ss could enhance alpha more easily than they could suppress it.

(2) Alpha control, defined as the difference in alpha level during alpha $O N$ and alpha OFF sessions, is possible without the feedback signal (following initial alpha feedback training).

(3) Tone-on alpha present and tone-off alpha present are equally effective kinds of feedback.

(4) There is no significant correlation between $\mathrm{HR}$ and percent alpha during alpha training.

(5) Alpha control is positively correlated with the amount of feedback provided, which is 
determined by the threshold of the feedback circuit.

\section{REFERENCES}

ADRIAN, E, D, \& MATHEWS, B, H. C. The Berger rhythm: Potential changes from the occipital lobes in man. Brain, 1934, 57, 355-385.

ANDERSEN, P.. \& ANDERSSON, S. Physiological basis of the alpha rhythm. New York: Appleton-Century-Crofts, 1968.

BROWN, B. Recognition of aspects of consciousness through association with electroencephalographic alpha activity represented by a light signal. Psychophysiology, 1970, 6, 442-453.

CLEELAND, C., BOOKER, H., \& HOSOKAWA, $K$. Alpha enhancement Due to feedback or the nature of the task? Paper presented to meeting of Society for Psychophysiological Research, New Orieans, November 1970 . KREITMAN, N, \& SHAW, J. Experimenta enhancement of alpha activity Electroencephalography \& Clinical Neurophysiology, 1965, 18, 147-155.

LACEY, J. I. Somatic response patterning and stress: Some revisions of activation theory. In M. Appley and $R$. Trumbull (Eds.), Psychological stress: Issues in $r e s e a r c h . \quad N$ e $\quad Y$ o r k : Appleton-Century-Crofts, 1967

LYNCH, J. J., ORNE, M T PASKEWITZ D., \& COSTELLO, J. An analysis of the feedback control of alpha activity. Paper presented to the Pavlovian Society of North America, Princeton, October 1969

NOWLIS, D \& KAMIYA.J The control of electroencephalowraphic alpha rhythms through auditory feedback and the associated mental activity. Psychophysiology, 1970, 6, 476-484.

NOTE

1. Data for SLM, Day 1, was not included because he closed his eyes during BL, giving inordinately high alpha levels.

\section{CURRENT LITERATURE ON ELECTRICAL RECORDING \& BFHAVIOR}

ADOLPH, A. R. (Neurophysiology Laboratory, Retina Foundation, 20 Staniford Street, Boston, Mass. 02114). Discrete slow potentials and threshold-level spikes in the Limulus ommatidium. Vision Research, 1971, 11, 371-376.

FUKADA, Y. (Research Group on Auditory and Visual Information Processing, NHK Broadcasting Science Research Laboratories, Setagaya-ku, Tokyo, Japan), \& SAIT, H. The relationship between response characteristics to flicker stimulation and receptive field organization in the cat's optic nerve fibers. Vision Research, 1971, $11,227-240$.

GRAHAM, N. (Rockefeller University, New York, N.Y. 10021), \& NACHIMIAS, J. Detection of grating patterns containing two spatial frequencies: $A$ comparison of single-channel and multiple-channels models. Vision Research, 1971, 11, 251-259.

HAMASAKI, D. I. (Bascom Palmer Eye Institute, Post Office Box 875, Biscayne Annex, Miami, Fla.
33152), \& STRECK, P. Properties of the Epiphysis cerebri of the small-spotted dogfish shark, Scyliorhinus caniculus L. Vision Research, 1971, 11, 189-198.

INGLE, D. (Neuropsychology Laboratory, McLean Hospital, Belmont, Mass. 02178). A possible behavioral correlate of delayed retinal discharge in anurans. Vision Research, 1971, 11, 167-168.

JEANNEROD, M. (Laboratoire de Médicine Expérimentals, Faculté de Médicine, Lyon, France), \& Sakai, K. Vision Research, 1971, 11, 161-165.

PADMOS, P. (Institute for Perception R V O-T N O, Kam pweg 5 , Soesterberg, The Netherlands), \& VAN NORREN, D. Cone spectral sensitivity and chromatic adaptation as revealed by human flicker-electroretinography. Vision Research, 1971, 11, 27-42.

PRESTRUDE, A. M. (Virginia Polytechnic Institute, Blacksburg, Va. 24061). Visual latencies at photopic levels of retinal illuminance. Vision Research, 1971,
11, 351-361.

REGAN, D., \& SPERLENG, H. G. (Department of Neural Sciences, University of Texas Graduate School of Biomedical Sciences, Texas Medical Center, Houston, Tex. 77025). A method of evoking contour-specific scalp potentials by chromatic checkerboard patterns. Vision Research, 1971, 11, 173-176.

WOOLSEY, C. N. (Laboratory of Neurophysiology, University of Wisconsin, Madison, Wis. 53706), CARLTON, T. G., KAAS, J. H., \& EARLS, F. J. Projection of visual field on superior colliculus of ground squirrel (Citellus tridecemlineatus). Vision Research, 1971, $11,173-176$.

ZANEN, A. (Brain Research Unit, University of Brussels, 115, Boulevard de Waterloo, 1000 -Brussels, Belgium), \& DEBECKER, J. Visual pigments and melanin contributions to the fast photovoltage of the human eye. Vision Research, 1971, 11, 169-172. 